\title{
Profiles of Patients Who Use Marijuana for Inflammatory Bowel Disease
}

\author{
Ann Marie Kerlin ${ }^{1} \cdot$ Millie Long ${ }^{2} \cdot$ Michael Kappelman ${ }^{2} \cdot$ Christopher Martin ${ }^{2} \cdot$ Robert S. Sandler ${ }^{2}$
}

Received: 26 March 2017 / Accepted: 23 March 2018 / Published online: 29 March 2018

\begin{abstract}
Background Marijuana is legal in a number of states for indications that include inflammatory bowel diseases (IBD), and patients are interested in its potential benefits.

Aims We aimed to describe the legal use of marijuana in individuals with IBD in the USA who participate within the CCFA Partners internet-based cohort.

Methods A total of 2357 participants who lived in states where prescription or recreational marijuana was legal, were offered the opportunity to complete a survey on marijuana use and IBD symptoms including perceived benefits of therapy. Bivariate statistics and logistic regression models were used to determine factors associated with marijuana use.

Results Surveys were completed by 1666 participants (71\%) with only $214(12.8 \%)$ indicating they had asked their medical doctor about its use and 73 actually using prescribed marijuana (4.4\%). Within the respondent group $(N=1666), 234$ participants lived where both medical and recreational marijuana is legal and $49(20.9 \%)$ reported recreational marijuana use specifically for IBD. Users reported positive benefits (80.7\%), but users also reported more depression, anxiety, pain interference, and lower social satisfaction than non-users. Those prescribed marijuana reported more active disease, and more use of steroids, narcotics, and zolpidem.

Conclusions Few IBD patients consulted their medical doctors about marijuana use or used prescription marijuana. Where recreational marijuana was available, usage rates were higher. Users reported benefits but also more IBD symptoms, depression, anxiety, and pain. Marijuana use may be higher in patients with IBD symptoms not well treated by conventional medical approaches.
\end{abstract}

Keywords Marijuana $\cdot$ Ulcerative colitis $\cdot$ Crohn's disease $\cdot$ Cannabis $\cdot$ Cannabinoids

\section{Introduction}

Crohn's disease (CD) and ulcerative colitis (UC) are chronic inflammatory bowel diseases (IBD) that significantly impair quality of life. There are increasing numbers of options for medical treatment of inflammation, including novel classes

Electronic supplementary material The online version of this article (https://doi.org/10.1007/s10620-018-5040-5) contains supplementary material, which is available to authorized users.

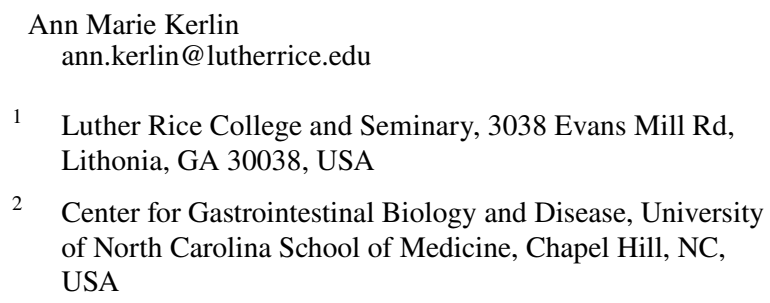

2 Center for Gastrointestinal Biology and Disease, University of North Carolina School of Medicine, Chapel Hill, NC, USA

of immunosuppressive and biologic therapies [1]. However, symptoms can remain despite treatment. Many patients have an interest in alternative therapies due to concerns about risks or side effects of medications, or as a supplement to standard treatment protocols. For these reasons, there has been interest in the patient and provider communities in understanding the role of marijuana in IBD.

A review of the literature conducted in 2015 concluded that more research was needed to determine whether there were any medical benefits of marijuana [2]. Both epidemiologic data and small trials demonstrate a possible role for cannabinoids in the symptomatic treatment of IBD. As of yet, it is unclear whether these therapies have anti-inflammatory effects, or whether they are masking the debilitating symptoms of ongoing inflammation in IBD [3].

A number of published studies suggest that patients with IBD who use marijuana can derive symptomatic 
benefits [4-7]. It is also apparent that a large number of IBD patients have tried marijuana, particularly older men [8]. There are limited data on the characteristics of marijuana users in the IBD population. In particular, further information is needed on disease activity, concomitant psychosocial factors, and whether IBD medications are also used by this population.

To learn more about the use of marijuana in the USA, we developed a survey to ascertain the characteristics of marijuana users in states where marijuana is legal by prescription or for recreational use. We hypothesized that few IBD patients would have access to prescribed marijuana, and we were interested in learning what benefits those using prescription or recreational marijuana might perceive.

\section{Materials and Methods}

CCFA Partners is a longitudinal, internet-based cohort of self-identified adult IBD patients [9] including nearly 15,000 patients who completed a baseline health survey and provide updates every 6 months. The present study was made available to patients residing in 25 states where prescription marijuana is legal; 4 of these states also legalized recreational marijuana use. The survey (see supplementary Fig. 1) was offered to 2357 participants over an 8-month time period. Of these, 1666 actually completed the survey $(65.7 \%$ response rate) and are included in the analysis.

We measured disease activity using the Short Crohn's Disease Activity Index; an sCDAI of less than 150 was defined as remission [10]. Using the Simple Clinical Colitis Activity Index (SCCAI) for ulcerative colitis or indeterminate colitis (UC/IC), remission was defined as an SCCAI of 2 or less [11].

The Short Inflammatory Bowel Disease Questionnaire (SIBDQ) is a measure of health-related quality of life that has been validated for use in IBD patients [12]. It consists of 10 questions rated on a seven-point Likert scale, with a test-retest reliability coefficient established at 0.65 , and Cronbach's alpha at 0.78 [12]. The higher the SIBDQ score, the better the measured quality of life (QOL).

We used several PROMIS ${ }^{\circledR}$ instruments to measure anxiety, depression, pain, sleep, and social satisfaction. PROMIS $^{\circledR}$ (Patient-Reported Outcomes Measurement Information System) instruments have undergone rigorous development and validation [13]. They are calibrated using a T-score metric with the mean of the US general population equal to 50 and SD equal to 10. Minimal important differences (MIDs) of these PROMIS domains for chronic diseases are in the range of $2-6[14,15]$. Higher scores indicate more of the domain being measured.

\section{Statistical Analysis}

All analyses were performed using SAS version 9.3 (SAS Institute, Cary, NC). Categorical variables were compared using Chi-square statistics. Continuous variables were compared using $\mathrm{t}$ tests or Wilcoxon rank-sum test as appropriate. For PROMIS domains, measures were dichotomously defined as PROMIS $t$ scores $\geq 55$, as this one-half standard deviation difference met criteria for minimal important differences. We used logistic regression modeling to determine independent predictors of marijuana use among IBD patients. Statistical significance was defined as a $p$ value less than 0.05 .

\section{Ethical Considerations}

Participants sign informed consent when they join the CCFA Partners cohort. The study was approved by the Institutional Review Board at the University of North Carolina.

\section{Results}

For participants living in states where medical marijuana was legal, only 214 participants $(N=1666,12.8 \%)$ reported that they had asked their medical doctor about its use for IBD symptoms. Eighty-three participants were prescribed marijuana and 73 actually used the drug (4.4\%). Among the 1666 respondents, 234 lived in states where both medical and recreational marijuana were legal and $49(20.9 \%)$ stated they used recreational marijuana specifically for their IBD. There were a total of 122 reports of legal marijuana use specifically for IBD; however, 8 participants reported both recreational and medical marijuana use, yielding a total of 114 unique participant responses.

The majority of marijuana users in this survey (80.7\%) perceived benefits of its use (presented in Table 1). These included improvement in pain (68\%), appetite (49\%), anxiety (48\%), fatigue (26\%), stool frequency (23\%), weight gain (20\%), and blood in stool (5\%), and there were two write-in responses indicating benefit for nausea (1.8\%). Twenty-two (19\%) users indicated no symptom relief.

Results comparing those patients who used prescribed recreational marijuana $(n=114)$, compared to non-users $(n=1552)$, on the PROMIS measures are presented in Table 2. Overall, those using marijuana reported significantly more anxiety, depression, pain, and lower social satisfaction.

We were interested in examining the characteristics of patients who were prescribed marijuana by their physicians to shed light on why physicians thought their patients might 
Table 1 Reported benefits of marijuana on IBD symptoms in CCFA partners among medical and recreational users

\begin{tabular}{lccc}
\hline Symptom improvements & $\begin{array}{l}\text { Used by prescription } \\
(N=73) n(\%)\end{array}$ & $\begin{array}{l}\text { Used recreationally } \\
(N=49) n(\%)\end{array}$ & $\begin{array}{l}\text { Total unique } \\
\text { responses }{ }^{\mathrm{a}}(N=114) \\
n(\%)\end{array}$ \\
\hline Pain & $56(76.7)$ & $27(55.1)$ & $78(68.4)$ \\
Fatigue & $18(24.7)$ & $15(30.6)$ & $30(26.3)$ \\
Appetite & $46(63.0)$ & $15(30.6)$ & $56(49.1)$ \\
Anxiety & $40(54.8)$ & $20(40.8)$ & $55(48.2)$ \\
Weight gain & $11(15.1)$ & $5(10.2)$ & $23(20.2)$ \\
Stool frequency & $19(26.0)$ & $5(10.2)$ & $23(20.2)$ \\
Blood in stool & $6(8.2)$ & $1(2.0)$ & $6(5.3)$ \\
Other & $21(28.8)$ & $10(20.4)$ & $30(26.3)$ \\
Did not help & $10(13.7)$ & $14(8.6)$ & $22(19.3)$ \\
\hline
\end{tabular}

a 8 participants were in both prescribed and recreational categories
Table 2 PROMIS measures in IBD patients using/not using marijuana

\begin{tabular}{lllc}
\hline & $\begin{array}{l}\text { Use of } \\
\text { marijuana } \\
(N=114)^{\mathrm{a}} \\
\text { Mean }(\mathrm{SD})\end{array}$ & $\begin{array}{l}\text { Non-use }(N=1552) \\
\text { Mean }(\mathrm{SD})\end{array}$ & $p$ value \\
\hline Anxiety & $54.1(9.8)$ & $51.0(9.2)$ & $<0.0001$ \\
Depression & $52.3(9.8)$ & $49.1(8.7)$ & $<0.0001$ \\
Pain interference & $55.5(10.4)$ & $50.1(9.5)$ & $<0.0001$ \\
Sleep disturbance & $52.3(3.2)$ & $52.1(3.3)$ & 0.30 \\
Social satisfaction & $48.0(10.4)$ & $50.9(9.7)$ & 0.0001 \\
\hline
\end{tabular}

PROMIS measures are reported using a T-score metric with the mean of the US general population equal to 50 and SD in the general population equal to 10

a 73 patients used prescribed marijuana and 49 used recreational marijuana; 8 participants were in states where both prescribed and recreational marijuana are legal and are counted only once

benefit from its use. There were 83 who were given prescriptions, and their characteristics are presented in Table 3. Most of the CD patients had active disease (57.4\%), as did UC/IC patients $(70.6 \%)$. Prescribed marijuana patients were also much more likely to have had IBD surgery (14.5 vs. $4.7 \%$, $p=0.0008)$, report a lower quality of life on the SIBDQ ( 4.5 vs. $5.3 ; p=0.0001)$, and were taking steroids ( 18.1 vs. $10.4 \%, p=0.04)$, narcotics (27.7 vs. $6.4 \%, p=0.0001)$, and zolpidem, a prescription sleep aid (12.0 vs. $4.0 \%, p=0.003)$. They were less likely to take aminosalicylates (22.9 vs. $36.9 \%, p=.01)$.

\section{Discussion}

The number of CCFA Partners participants who reported being prescribed marijuana or using it specifically for IBD were few $(n=114,6.8 \%)$, as we hypothesized. This low per-centage, compared to Weiss and Freidenberg's findings of
$67.3 \%$ ever use of marijuana and hashish in IBD patients in a large population study [8], may be due to the fact that our survey was not anonymous, since participants are part of an ongoing longitudinal study. We also asked about legal marijuana use specifically for IBD, rather than any use ever.

When exploring the profiles of patients using marijuana, the majority stated it was helpful with symptoms (86\%), particularly identifying its benefits for pain, appetite, and anxiety, followed by fatigue, weight gain, stool frequency, and blood in stool. Four other studies found similar results, especially identifying pain. Allegretti et al. [4] found that patients reported benefits for pain, appetite, nausea, and diarrhea $(N=292)$. In a study by Storr et al. [7] $(N=303)$ marijuana was perceived helpful for abdominal pain, abdominal cramping, joint pain, and diarrhea, but patients had a higher risk of IBD surgery; our findings are similar. Lal et al. [6] $(N=191)$ found that pain, diarrhea, and appetite were the main symptom improvements reported. In a retrospective observational study, Naftali et al. [16] $(N=30)$ reported multiple benefits including objective markers of disease in male CD patients. All four studies, and ours, reveal somewhat different findings.

Two clinical trials were located in the literature. Naftali et al. [5] found a clinical response in 10/11 patients and full remission in 5 of those patients, when using smoked marijuana in their placebo-controlled clinical trial. Another placebo-controlled clinical trial $(n=65)$ by de Vries et al. [17] found no difference between placebos and orally ingested tablets containing tetrahydrocannabinol used for pain control. Some differences in methods of administration and dosing may contribute to these conflicting results.

Because the medical marijuana users in our study reported more prior surgeries, and a poorer quality of health overall, it is conjectured that patients who are prescribed marijuana may have disease that is poorly controlled with conventional measures or that they may have significant symptoms that are not addressed by conventional therapies 
Table 3 Characteristics of study participants who were prescribed marijuana by a physician

\begin{tabular}{|c|c|c|c|}
\hline \multirow[t]{2}{*}{ Characteristics } & \multicolumn{2}{|c|}{ Medical marijuana } & \multirow[t]{2}{*}{$p$ value* } \\
\hline & $\begin{array}{l}\text { Prescribed by } \\
\text { MD }(n=83)\end{array}$ & $\begin{array}{l}\text { Not } \\
\text { prescribed } \\
(n=1583)\end{array}$ & \\
\hline \multicolumn{4}{|l|}{ Demographics } \\
\hline Female ( $\%$ yes) & 67.5 & 69.9 & 0.62 \\
\hline Age (SD) & $41.5(14.6)$ & $44.1(15.1)$ & 0.12 \\
\hline \multicolumn{4}{|l|}{ Disease characteristics (\%) } \\
\hline $\mathrm{CD}^{\mathrm{a}}$ & 71.1 & 62.3 & 0.12 \\
\hline $\mathrm{UC} / \mathrm{IC}^{\mathrm{b}}$ & 28.9 & 37.7 & \\
\hline In remission, overall & 39.1 & 62.9 & 0.0002 \\
\hline $\mathrm{CD}(\mathrm{sCDAI} \text { remission })^{\mathrm{c}}$ & 42.6 & 68.0 & 0.0007 \\
\hline UC/IC (SCCAI remission) ${ }^{\mathrm{d}}$ & 29.4 & 54.0 & 0.05 \\
\hline $\begin{array}{l}\text { Time since diagnosis; years } \\
\text { (SD) }\end{array}$ & $12.5(11.7)$ & $14.5(13.1)$ & 0.17 \\
\hline History of IBD surgery & 14.5 & 4.7 & 0.0008 \\
\hline SIBDQ $^{\mathrm{e}}$ & $4.5(1.2)$ & $5.3(1.1)$ & $<0.0001$ \\
\hline \multicolumn{4}{|l|}{ Physician characteristics } \\
\hline $\begin{array}{l}\text { Primary care physician (\% } \\
\text { yes) }\end{array}$ & 94.0 & 91.7 & 0.68 \\
\hline \multicolumn{4}{|l|}{ GI setting (\%) } \\
\hline University/academic setting & 28.9 & 21.8 & 0.21 \\
\hline Private practice & 56.6 & 67.2 & \\
\hline Other & 14.5 & 11.0 & \\
\hline \multicolumn{4}{|l|}{ Current IBD medications (\%) } \\
\hline Steroids & 18.1 & 10.4 & 0.04 \\
\hline Biologics & 43.4 & 42.7 & 0.91 \\
\hline Immunomodulators & 20.5 & 27.1 & 0.20 \\
\hline Aminosalicylates (5-ASA) & 22.9 & 36.9 & 0.01 \\
\hline Narcotics & 27.7 & 6.4 & $<0.0001$ \\
\hline \multicolumn{4}{|l|}{ Psychotropic medications (\%) } \\
\hline SNRIs $^{\mathrm{f}}$ & 8.4 & 5.1 & 0.20 \\
\hline SSRIs ${ }^{g}$ & 8.4 & 7.9 & 0.82 \\
\hline Benzodiazepines & 14.5 & 10.2 & 0.20 \\
\hline Tricyclic antidepressants & 1.2 & 0.6 & 0.40 \\
\hline Zolpidem & 12.0 & 4.0 & 0.003 \\
\hline Buspirone & 0.0 & 0.6 & 1.00 \\
\hline
\end{tabular}

Numbers are percents unless otherwise indicated

* $p$ value by Chi square

${ }^{a}$ Crohn's disease

${ }^{\mathrm{b}}$ Ulcerative colitis/indeterminate colitis

'Short Crohn's disease activity index

${ }^{\mathrm{d}}$ Simple clinical colitis activity index

${ }^{\text {e }}$ Short Inflammatory Bowel Disease Questionnaire

${ }^{\mathrm{f}}$ Serotonin-norepinephrine reuptake inhibitors

${ }^{\mathrm{g}}$ Selective serotonin reuptake inhibitors (such as visceral hypersensitivity), and more psychosocial comorbidities.

There have been recent studies that found positive effects of cannabis on IBD in animal studies [18-20], and articles demonstrating positive effects of cannabinoids at the molecular level [21-24]. Cannabis can reduce cramping and slow GI motility [25]. Exogenous cannabinoids can also reduce colitis [25]. There are also questions in regard to the safety profile of marijuana, particularly in regard to dosing and route of administration to maximize benefits and reduce harms [26]. Physicians should be aware of the limited data supporting marijuana use and follow AMA guidelines and legal restrictions [26].

There are limitations to this study. We did not capture methods of administration or dosing, and this makes comparisons with other surveys problematic. We also do not have the time frame between when prescription marijuana users were diagnosed and when they began using marijuana, which might help determine its effects on the course of disease, for instance. Additionally, we did not capture perceived adverse events from marijuana use. Finally, the measures of disease activity used in CCFA Partners, while validated in self-report, are symptom-based. Therefore, we were unable to define disease activity through objective markers of inflammation, such as C-reactive protein or endoscopic measures of inflammation.

While legal marijuana use was limited in our survey, users perceived some benefit. Marijuana may be filling an unmet medical need for some patients. Ultimately, large-scale clinical trials are needed to clarify mixed results found in the current literature.

Funding This research was supported, in part, by Grants from the Crohn's and Colitis Foundation of American and by the National Institutes of Health P30 DK034987.

\section{Compliance with ethical standards}

Conflict of interest The authors declare no conflicts of interest.

\section{References}

1. Ananthakrishnan A, Kwon J, Raffals L, et al. Variation in treatment of patients with inflammatory bowel diseases at major referral centers in the United States. Clin Gastroenterol Hepatol. 2015;13:1197-1200.

2. Belendiuk K, Baldini L, Bonn-Miller M. Narrative review of the safety and efficacy of marijuana for the treatment of commonly state-approved medical and psychiatric disorders. Addict Sci Clin Pract. 2015;10:1-10.

3. Ahmed W, Katz S. Therapeutic use of cannabis in inflammatory bowel disease. Gastroenterol Hepatol. 2016;12:668-679.

4. Allegretti J, Courtwright A, Lucci M, Korzenik J, Levine J. Marijuana use patterns among patients with inflammatory bowel disease. Inflamm Bowel Dis. 2013;19:2809-2814. 
5. Naftali T, Schleider B, Dotan I, Lansky E, Benjaminov F, Konikoff $F$. Cannabis induces a clinical response in patients with Crohn's disease; a prospective placebo-controlled study. Clin Gastroenterol Hepatol. 2013;11:1276-1280.

6. Lal S, Prasad N, Ryan M, et al. Cannabis use amongst patients with inflammatory bowel disease. Eur J Gastroenterol Hepatol. 2011;23:891-896.

7. Storr M, Devlin S, Kaplan G, Panaccione R, Andrews C. Cannabis use provides symptom relief in patients with inflammatory bowel disease but is associated with worse disease prognosis in patients with Crohn's disease. Inflamm Bowel Dis. 2014;20:472-480.

8. Weiss A, Friedenberg F. Patterns of cannabis use in patients with inflammatory bowel disease: a population based analysis. Drug Alcohol Depend. 2015;156:84-89.

9. Long M, Kappelman M, Martin C, et al. Development of an internet-based cohort of patients with inflammatory bowel disease (CCFA partners); Methodology and initial results. Inflamm Bowel Dis. 2012;18:2099-2106.

10. Thia K, Faubion W, Loftus E, Persson T, Persson A, Sandborn W. Short CDAI: development and validation of a shortened and simplified Crohn's disease activity index. Inflamm Bowel Dis. 2011;17:105-111.

11. Jowett S, Seal C, Phillips E, Gregory W, Barton J, Welfare M. Defining relapse of ulcerative colitis using a symptom-based activity index. Scand J Gastroenterol. 2003;38:164-171.

12. Irvine $\mathrm{E}$, Zhou Q, Thompson $\mathrm{A}$. The short inflammatory bowel disease questionnaire; a quality of life instrument for community physicians managing inflammatory bowel disease. CCRPT investigators. Canadian Crohn's Relapse Prevention Trial. Am J Gastroenterol. 1996;91:1571-1578.

13. Cella D, Yount S, Rothrock N. The patient-reported outcomes measurement information system (PROMIS): progress of an NIIH roadmap cooperative group during tis first two years. Med Care. 2007;45:S3-S11.

14. Kappelman M, Long M, Martin C, et al. Evaluation of the patientreported outcomes measurement information system in a large cohort of patients with inflammatory bowel diseases. Clin Gastroenterol Hepatol. 2014;12:1315-1323.
15. Yost K, Eton D, Garcia S, Cella D. Minimally important differences were estimated for six patient-reported outcomes measurement information system-cancer scales in advanced-stage cancer patients. J Clin Epidemiol. 2011;64:507-516.

16. Naftali T, Lev LB, Yablecovitch D, Half E, Konikoff FM. Treatment of Crohn's disease with cannabis: an observational study. Isr Med Assoc J. 2011;13:455-458.

17. de Vries M, van Rijckevorsel D, Vissers K, Wilder-Smith O, van Goor $\mathrm{H}$. Tetrahydrocannabinol does not reduce pain in patients with chronic abdominal pain in a phase 2 placebo-controlled study. Clin Gastroenterol Hepatol. 2017;15:1079-1086.

18. Massa F, Marsicano G, Hermann H, et al. The endogenous cannabinoid system protects against colonic inflammation. Mucosal Immunol. 2011;4:574-583.

19. Borrelli F, Fasolino I, Romano B, et al. Beneficial effect of the non-psychotropic plant cannabinoid cannabigerol on experimental inflammatory bowel disease. Biochem Pharmacol. 2013;85:1306-1316.

20. Borrelli F, Aviello G, Romano B, et al. Cannabidiol, a safe and non-psychotropic ingredient of the marijuana plant Cannabis sativa, is protective in a murine model of colitis. $\mathrm{J} \mathrm{Mol} \mathrm{Med}$. 2009;87:1111-1121.

21. Storr M, Keenan C, Zhang H, Patel K, Makriyannis A, Sharkey K. Activation of the cannabinoid 2 receptor (CB2) protects against experimental colitis. Inflamm Bowel Dis. 2009;15:1678-1685.

22. Kunos G, Pacher P. Cannabinoids cool the intestine. Nat Med. 2008;10:678-679.

23. Wright K, Duncan M, Sharkey K. Cannabinoid CB2 receptors in the gastrointestinal tract: a regulatory system in states of inflammation. Br J Pharmacol. 2008;153:263-270.

24. Izzo A, Sharkey K. Cannabinoids and the gut: new developments and emerging concepts. Pharmacol Ther. 2010;126:21-38.

25. Sharkey K, Wiley J. The role of the endocannabinoid system in the brain-gut axis. Gastroenterology. 2016;151:252-266.

26. Chaudhry H, Hengerer A, Snyder G. Medical board expectations for physicians recommending marijuana. J Am Med Assoc. 2016;316:577-578. 